\title{
ON CONNECTIVITY PROPERTIES OF FINITE-DIMENSIONAL GROUPS
}

\author{
SIGMUND N. HUDSON
}

1. Introduction. Gleason and Palais [5] have shown that (Theorem A) a locally arcwise connected, finite-dimensional topological group is locally compact and thereby a Lie group. The key lemma in the proof of this theorem is a lemma of Gleason [17] and Montgomery [18], as follows: (Lemma A) let $G$ be a locally arcwise connected topological group, and let $X$ be a nonvoid compact, metrizable subspace of $G$ of dimension $n<\infty$; then either $X$ has an interior point or else there is an $\operatorname{arc} A$ in $G$ such that $A X$ is compact, metrizable, and of dimension greater than $n$. These theorems are significant in that the usual assumptions of local compactness have been replaced by local arcwise connectivity assumptions. This paper continues the study of connectivity properties of topological groups which are not locally compact. The main result is that a locally connected, arcwise connected, finite-dimensional group is a Lie group, thereby providing another solution to a "modified Hilbert's Fifth Problem" (see [12] or [6]). Other versions of the Gleason, Palais, and Montgomery results are then derived, with the result that local arcwise connectivity may be replaced by local connectivity plus arcwise connectivity.

2. If $J$ is a topology for a set $X$, then $\mathfrak{T}(J)$ is defined to be the topology for $X$ which has as base the arcwise connected components of open sets in J. If $G$ is a topological group with topology $J$, then $(G, \mathfrak{M}(J))$ is locally arcwise connected and the identity function $i:(G, \mathfrak{M}(\mathfrak{J})) \rightarrow(G, \mathfrak{J})$ is continuous. We call $\mathcal{G}=(G, \mathfrak{M}(\mathfrak{J}))$ the locally arcwise connected covering group of $G$. If $G$ is arcwise connected, then $\mathcal{G}$ is connected. See Gleason and Palais [5, p. 634] or Young [16] for details.

For a topological group $G$, we $\operatorname{define} \operatorname{dim} G$ to be $\operatorname{lub}\{\operatorname{cd}(C): C$ is a compact subset of $G\}$, where $\operatorname{cd}(C)$ denotes the cohomological dim of $C$ due to Cohen [2]. Using this dimension function and AlexanderCech cohomology, the proof by Gleason and Palais of Lemma A (and hence Theorem A) can be modified so as to eliminate the metrizability assumption and so that "finite-dimensional" is taken with respect to $\operatorname{dim} G$ just defined. These modifications use for the most part the Eilenberg-Steenrod axioms, the Map Excision Theorem, and the Roof Theorem of A. D. Wallace.

Received by the editors January 30, 1969. 
The locally arcwise connected covering group can be a useful tool in elementary Lie group theory. For example, Gleason [17] has shown how this covering group may be used to prove Yamabe's theorem that an arcwise connected subgroup $G$ of a Lie group $L$ is an analytic subgroup. For, by Theorem A, the locally arcwise connected covering

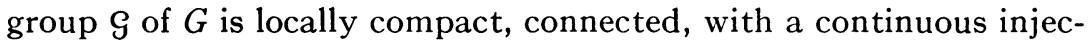
tion into a Lie group; and by Cartan and Chevalley's theorem $[1$, p. 130] is a Lie group. Also, using the definition of a Lie subgroup $S$ of a Lie group $G$ adopted by some authors (see [3], [14], [7], [11]), any subgroup $S$ of a Lie group $L$ is a Lie subgroup if one uses the locally arcwise connected covering group of $S$ with respect to the relative topology on $S$. The discrete topology for $S$ could also be used.

The main result is now given. Examples of arcwise connected, finite-dimensional groups which might be kept in mind are (a) a dense one-parameter subgroup of the $n$-dimensional abelian, toral group. Also there is (b) the group $(R, J)$ of Omori [13] obtained as follows: an "extraordinary" topology $\mathcal{S}$ on the integers $Z$ is defined such that $Z$ is a topological group and there is a countable base $\left\{U_{n}\right\}$ of neighborhoods at $0 \in Z$, each $U_{n}$ containing an infinite number of integers. After defining $V_{n}$ to be the open interval $\left(-2^{-n}, 2^{-n}\right)$ in the real numbers $R, W_{n, k}$ is defined to be $U_{n}+V_{k}$. Then $\left\{W_{n, k}\right\}$ becomes a countable neighborhood base at 0 for the topology $J$ on $R$. Example (c) is obtained from example (b) by using a connected Lie group for which there is a discrete, countably infinite subgroup $D$ contained in the center of $G$. By using an "extraordinary" topology on $D$ and an open ball at $e$ in $G$, one constructs a topology on $G$. See Hewitt and Ross [8, p. 27] for another "extraordinary" topology on $Z$. A typical open set of the groups in examples (a)-(c) may be described roughly and geometrically as composed of arcwise connected regions distributed throughout some underlying manifold space associated with each group.

TheOREM 1. Let $G$ be a locally connected, arcwise connected group with $\operatorname{dim} G<\infty$. Then $G$ is a Lie group.

Proof. The locally arcwise connected covering group $\mathcal{G}$ of $G$ is a

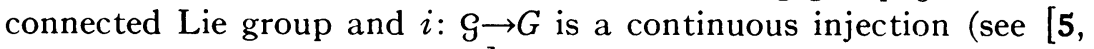
Corollary 7.3 and Theorem 3.2]). It will be shown that some compact neighborhood $V$ of the identity $e$ in $G$ is also a neighborhood of the identity in $G$, thereby implying that the identity function is open and a homeomorphism. Let $V$ be a compact, symmetric neighborhood of $e$ in $\mathrm{g}$. Then $V^{3}$-Int $V$ (with respect to $\mathrm{G}$ ) is compact in both $\mathrm{G}$ and $G$, hence closed in $G$. Let $W$ be a connected, symmetric neighbor- 
hood of $e$ in $G$ such that $W^{2} \cap\left(V^{3}-\right.$ Int $\left.V\right)=\varnothing$. We assume by way of contradiction that $W$ is not contained in Int $V$; if it were then $V$ would be a neighborhood of $e$ in $G$. We choose $u \in W$-Int $V$. Then $u \notin V^{3}$ and $e \notin u V^{3}$. Compactness of $u V^{3}$ and complete regularity of $G$ imply the existence of a continuous real-valued $f: G \rightarrow R$ such that $f$ is zero on $(G-W) \cup u V^{3}, f(e)=1$, and $f \geqq 0$. Define $h: G \rightarrow R$ by $h(x)$ $=\sup \{f(x v): v \in V\}$. Then $h$ is continuous, $h \geqq 0, h(u)=0, h(e) \geqq 1$. Since $h$ takes at least two values on the connected set $W, h$ takes uncountably many values on $W$.

We now show $h$ is constant on the sets $W \cap x V$, for $x \in g$. For $w_{1}$ and $w_{2} \in W \cap x V$, let $w_{i}=x v_{i}$ with $v_{i} \in V$; so $w_{1}^{-1} w_{2}=v_{1}^{-1} v_{2} \in V^{2}$ $\cap W^{2} \subset V$. Since the above supremum is attained on the compact $V$, $h\left(w_{2}\right)=f\left(w_{2} v_{3}\right)$. Either $w_{2} v_{3} \in G-W$ or not; so that either $h\left(w_{2}\right)=0$, or else $w_{1}^{-1} w_{2} v_{3} \in W^{-1} W \cap V V \subset$ Int $V$, so that $h\left(w_{1}\right) \geqq f\left(w_{1} w_{1}^{-1} w_{2} v_{3}\right)$ $=h\left(w_{2}\right)$. In both cases $h\left(w_{1}\right) \geqq h\left(w_{2}\right)$. By symmetry $h\left(w_{1}\right)=h\left(w_{2}\right)$.

Finally it is shown that $W$ is covered by at most countably many sets of the form $W \cap x V$ (for $x \in W$ ). It suffices to show that any pair of these sets are either equal or disjoint; because they are neighborhoods in a connected manifold, which can afford at most countably many such neighborhoods. Suppose $\left(W \cap x_{1} V\right) \cap\left(W \cap x_{2} V^{\prime}\right) \neq \varnothing$. If $z \in x_{1} V \cap x_{2} V \cap W$, then $z=x_{1} v_{1}=x_{2} v_{2}$, and $x_{1}^{-1} x_{2}=v_{1} v_{2}^{-1} \in W^{2} \cap V^{2} \subset V$. Hence $x_{2} \in x_{1} V, x_{2} V \subset x_{1} V V$, and $W \cap x_{2} V \subset W \cap x_{1} V^{2} \subset x_{1}\left(W^{2} \cap V^{2}\right)$ $\subset x_{1} V$. Hence $W \cap x_{2} V \subset W \cap x_{1} V$. Again by symmetry we are finished. So we have the contradiction that $h$ takes on countably and uncountably many values.

This proof is a vast simplification by an unknown referee of the original proof. The idea of using connectivity to obtain uncountably many objects and a connected manifold to obtain countably many was common to both proofs, but the original proof was burdened with excessively cumbersome machinery.

3 . We now give the promised analogue to Lemma A (see Theorem 4).

Theorem 2. Let $G$ be an arcwise connected group. Then $G$ is finitedimensional iff its locally arcwise connected covering group $G$ is finitedimensional.

Proof. First suppose that $g$ is finite-dimensional, so that $g$ is a connected Lie group by Theorem A of Gleason and Palais. Let $k$ be the dimension of this Lie group, and let $B$ be a compact $k$-cell containing $e \in g$. For any compact subspace $C$ of $G, B C$, as a subspace of $G$, is compact and has dimension at least $k$. But $B C$ is closed in $G$ and has dimension $k$ at each $x \in B C$. Hence $B C$ is the (at most) countable 
union of an increasing tower of compact $k$-dimensional subsets $D_{i} \subset \mathcal{G}, \mathcal{G}$ being a connected manifold. Hence $B C$ is the countable union of sets compact in $G$, by continuity of the identity function $i: \Im \rightarrow G$. Thus $C$ has dimension at most $k$. Thus the compact subsets $C$ of $G$ have dimensions bounded by $k$, and $G$ is finite-dimensional. The converse is easily proved.

THEOREM 3. If $G$ is a topological group, then $G$ is arcwise connected and finite-dimensional iff there exists a connected Lie group $L$ and a one-to-one, continuous, onto homorphism $f: L \rightarrow G$.

Proof. If $G$ is arcwise connected and finite-dimensional, use $\mathcal{G}$ and $i$. If there exists $L$ and $f$ as above, we use the following commutative diagram

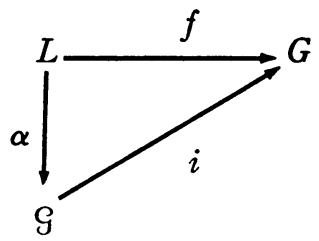

Define $\alpha=i^{-1} \circ f$. Then $\alpha$ is continuous by Theorem 3.2 and an isomorphism by Theorem 4.1 of [5]. Hence the previous Theorem 2 implies that $G$ is finite-dimensional.

THEOREM 4. Let $G$ be a locally connected, arcwise connected topological group. Let $C$ be a compact subspace of $G$ having dimension $k$. Then either the interior of $C$ is nonempty, or else there is an arc $I$ in $G$ such that $\operatorname{dim} C I>k$.

Proof. We may suppose $G$ is not finite-dimensional, for otherwise $G$ is a Lie group by Theorem 1 . Hence $\mathcal{G}$ is infinite-dimensional by Theorem 2. Let $C$ be a compact subset of $G$ having dimension $k$. We may apply the Gleason-Palais Lemma $A$ to the infinite-dimensional $\oint k+1$ times to obtain the existence of arcs $I_{1}, \cdots, I_{k+1}$ in $\oint$ such that $\operatorname{dim}\left(I_{1} \cdots I_{k+1}\right)>k$. For each $i$ with $1 \leqq i \leqq k+1$ let $\sigma_{i}$ be the mapping from $[0,1]$ onto $I_{i}$ defining the $\operatorname{arc} I_{i}$, and let $g$ be a continuous, onto mapping of $[0,1]$ to $[0,1]^{k+1}$. Then $i \circ m^{\prime} \circ\left(\sigma_{1} \times \cdots \times \sigma_{k+1}\right) \circ g$ is a mapping of $[0,1]$ onto $i\left(I_{1} \cdots I_{k+1}\right)$ (here $m^{\prime}$ is the multiplication function $m$ expanded to $k+1$ coordinates, $m$ continuous on $\mathcal{G}$ ). Hence $C i\left(I_{1} \cdots I_{k+1}\right)=C I$ has dimension at least $k+1$ for the arc $I \subset G$.

F. B. Jones [10] has given an example of a connected, locally connected subgroup of the vector group $R^{2}$ which contains no arcs and is 
dense in $R^{2}$. Snce this group is not a Lie group, the essential role of arcwise connectivity in the preceding theorems is displayed.

In the examples given preceding Theorem 1 a prominent discrete subgroup $D$ of a Lie group played an important role in defining a neighborhood base at the identity of the group in question. The author is not at present able to determine whether every finitedimensional, arcwise connected group $G$ has such a subgroup $D$ contained in its locally arcwise connected covering group $G$.

\section{REFERENCES} 1946.

1. C. Chevalley, Theory of Lie groups. I, Princeton Univ. Press, Princeton, N. J.,

2. Haskell Cohen, $A$ cohomological definition of dimension for locally compact Hasudorff spaces, Duke Math. J. 21 (1954), 209-224.

3. P. M. Cohn, Lie groups, Cambridge Univ. Press, Cambridge, 1961.

4. J. Dugundji, Topology, Allyn and Bacon, Boston, Mass., 1966.

5. A. Gleason and R. Palais, On a class of transformation groups, Amer. J. Math. 79 (1957), 631-648.

6. V. Gluškov, The structure of locally compact groups and Hilbert's fifth problem, Uspehi Mat. Nauk 12 (1957), no. 2 (74), 3-41=Amer. Math. Soc. Transl. (2) 15 (1960), 55-93.

7. S. Helgason, Differential geometry and symmetric spaces, Academic Press, New York, 1962.

8. E. Hewitt and K. Ross, Abstract harmonic analysis. I, Academic Press, New York, 1963.

9. K. Iwasawa, On some types of topological groups, Ann. of Math. (2) 50 (1949), 507-558.

10. F. Burton Jones, Connected and disconnected plane sets and the functional equation $f(x)+f(y)=f(x+y)$, Bull. Amer. Math. Soc. 49 (1942), 115-120.

11. S. Kobayashi and K. Nomizu, Foundation of differential geometry, Interscience, New York, 1963.

12. D. Montgomery and L. Zippin, Topological transformation groups, Interscience, New York, 1955.

13. H. Omori, Some examples of topological groups, J. Math. Soc. Japan 18 (1966), 147-153.

14. P. Tondeur, Introduction to Lie groups and transformation groups, SpringerVerlag, Heidelberg, 1965.

15. H. Yamabe, On an arcwise connected subgroup of a Lie group, Isaka Math. J. 2 (1950), 13-14.

16. G. S. Young, The introduction of local connectivity by change of topology, Amer. J. Math. 68 (1946), 479-494.

17. A. M. Gleason, Arcs in locally compact groups, Proc. Nat. Acad. Sci. U.S.A. 38 (1950), 663-667.

18. D. Montgomery, Theorems on the topological structure of locally compact groups, Ann. of Math. 50 (1949), 570-580.

Syracuse University 